\title{
Adaptive forward collision warning algorithm for automotive applications
}

\author{
SeyedMehrdad Hosseini, Nikolce Murgovski, Gabriel Rodrigues de Campos, Jonas Sjöberg
}

\begin{abstract}
This paper proposes an adaptive collision warning algorithm (CWA) that supports the driver by issuing early warnings for collision avoidance without noticeably increasing the risk of false alarms in real traffic. This algorithm can also detect when an emergency intervention is necessary. Compared to existing CWAs, the proposed solution in this paper triggers alarms by solving a linear convex program using traffic data, road's constraints and bicycle model dynamics, and by incorporating an adaptive (speed-dependent) warning threshold. A collision threat is detected by determining feasible steering trajectories without altering the vehicle's longitudinal velocity.
\end{abstract}

\section{INTRODUCTION}

Active safety functions are designed to warn and, if necessary, intervene in critical situations in order to help drivers avoiding accidents. Many advanced methods have been already implemented in road vehicles during the past few years. Some examples include emergency braking system (EBS), adaptive cruise control (ACC), lane keeping aid (LKA), lane change aid (LCA) and collision warning (CW) systems [1], [2], [3].

Collision warning algorithms (CWAs) play a particular important role in active safety since they are aimed at notifying drivers of upcoming threats and potential collisions. Such an argument is fuelled by alarming numbers indicating that American drivers remained passive before the collision in over $78 \%$ of rear-end crashes [2]. Furthermore, it is also reported in [2] that a forward collision warning system has the possibility of preventing $51 \%$ of the rear-end collisions.

Generally speaking, CWAs include a decision algorithm estimating potential threats and can, if necessary, warn the driver by activating visual and audio signals. In extreme cases, whenever the driver does not react and a collision is assessed to be unavoidable, an emergency intervention system may be triggered in an appropriate manner. Several assessment algorithms for forward collision warning systems have been proposed in literature. In general, these algorithms try to predict the future state of the involved vehicles in order to estimate the effort needed to avoid an accident [4], [5], [6].

\footnotetext{
The authors are with the Department of Signals and Systems, Chalmers University of Technology, Gothenburg, Sweden. \{mehrdad.hosseini, nikolce.murgovski, gabriel.campos, jonas.sjoberg\}@chalmers.se.

G. R. Campos is also with the DEIB, Politecnico di Milano, Italy. gabriel.rodriguesdecamposepolimi.it

This work is supported by VINNOVA through the project Non hit Car and Truck within the Fordonsstrategisk Forskning och Innovation (FFI) research program, grant AD14VARI02 and by the European Commission Seventh Framework Program under the project AdaptIVe, grant agreement number 610428. Financial support from the VINNOVA-FFI project A-drive is also gratefully acknowledged.
}

Previously proposed solutions range from rather simple methods, measuring the collision risk in terms of the time to collision (TTC) [7], the predicted minimum distance [8] or the required deceleration [9], to model predictive control [10] and path planning [11], [12]. Among others, [13] proposes a lane change maneuver formulated as an optimization problem with a point mass vehicle model (for fast computation), [14] presents an optimal trajectory generator minimizing the yaw acceleration, while [15], [16] show a collision warning system based on two meaningful indices, i.e., the steering threat number (STN) and braking threat number (BTN). In [16], the intervention threshold is modified by considering an appropriate model for a distracted driver.

However, the previously developed collision warning algorithms consider the vehicle as a point mass. Furthermore, warning systems can become annoying and useless if warnings are triggered regardless of vehicle's dynamics, physical limitations and an appropriate warning threshold.

In order to handle these issues, in this paper we propose a novel CWA for forward collision avoidance. Our solution relies on two main functions: the first function, which is related to the vehicle's and road's characteristics, generates a feasible trajectory and steering policy suitable for a safe lane change; the second, determines when to trigger an alarm signal by calculating an adaptive threshold with respect to the speed of the host and leading vehicles. The main objective of this paper is to present a systematic methodology of an adaptive CWA to avoid forward collisions. The main contribution relies on a novel optimization algorithm using a bicycle model for the vehicle dynamics, safety constraints and an adaptive threshold that allows a speed-dependent CWA. This increases the accuracy of the CWA with respect to the realistic constraints. It also provides earlier warnings regarding the speed of both vehicles without increasing the number of false alarms.

The paper is organized as follows. Section II presents the main concepts behind collision warning algorithms. In Section III, a conventional CWA is shown while, Section IV describes our novel solution. Simulation results comparing the typical and proposed CWA are discussed in Section V and conclusion and perspectives are finally presented in Section VI.

\section{COLLISION WARNING ALGORITHM}

A typical forward collision scenario is presented in Fig. 1. Ideally, whenever the vehicle gets dangerously close to the leading vehicle, alarms should be triggered based on the relative distance (range) and the relative velocity (range rate). In critical cases, whenever the driver is unwilling or 


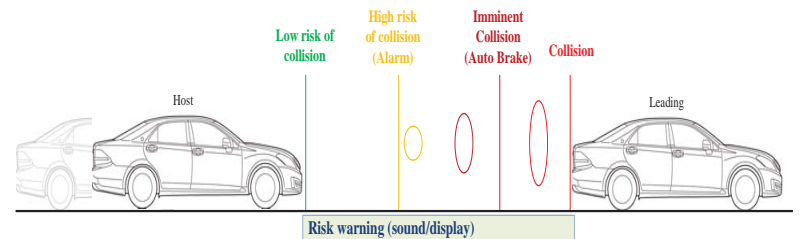

Fig. 1. Collision avoidance by steering or braking. When the host vehicle is getting close to the leading vehicle, the warning system will trigger an alarm provided that the relative distance exceeds a given threshold. After reaching a second threshold, at a shorter relative distance, the emergency braking system will commence to stop the vehicle before any colliding.

incapable of performing an evasive manoeuver, an emergency intervention should ultimately be triggered, such as, for example, an emergency braking by the EBS.

In the considered scenario, a collision is said to be imminent if it cannot be avoided by any feasible steering or braking actions. For forward collision situation, it is therefore possible to parameterize a collision based on the readings of the vehicles's lateral acceleration: when the maximum achievable lateral acceleration is lower than the requested value for a feasible lane change, safety can no longer be guaranteed [1], [17], [18].

The ratio between the requested lateral acceleration (due to steering) and the maximum achievable lateral acceleration is denoted as the steering threat number (STN). The STN has a key role in evaluating traffic threat situations [17] and is often used as a quantifier of the collision risk [1], [18]. Let TTC denote the expected time to collision, $a_{y_{h}}^{\text {req }}$ the requested lateral acceleration necessary for collision avoidance at TTC, and $a_{y_{h}}^{\max }$ the maximum achievable lateral acceleration of the host vehicle. Then, the STN can be defined as

$$
\mathrm{STN} \triangleq\left|\frac{a_{y_{h}}^{\text {req }}}{a_{y_{h}}^{\max }}\right| .
$$

It follows from the previous expression that if $\mathrm{STN} \leq 1$ the collision can be avoided, while otherwise the collision can no longer be avoided solely by performing a steering maneuver. Therefore, and emergency braking should be triggered, for instance. Note that while some methods exist to compute the requested lateral acceleration [12], [13], the maximum achievable lateral acceleration is normally considered as a fixed value [15].

\section{Conventional CWA}

This section describes a conventional CWA and provides a mathematical model for the requested acceleration and TTC, based on a point mass vehicle model.

1) Requested lateral acceleration: A method for calculating the requested acceleration is introduced in [1], for a lane change scenario where the host vehicle is subject to a constant lateral acceleration $a_{y_{h}}^{\text {req }}$ and constant longitudinal speed $v_{x_{h}}$, in the vehicle fixed coordinate system. In this method, the steering maneuver follows a circular path, as

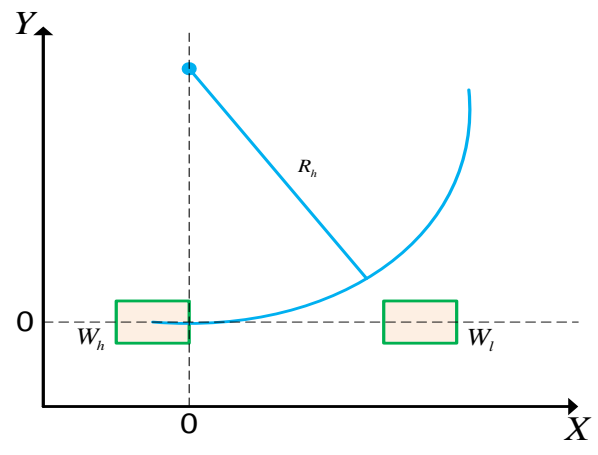

Fig. 2. A steering avoidance trajectory by constant lateral acceleration.

depicted in Fig. 2, with a radius

$$
R_{h}=\frac{v_{x_{h}}^{2}}{a_{y_{h}}^{\text {req }}} .
$$

Assuming that the center of gravity of the host vehicle passes through the origin, the trajectory swept by the left side of the vehicle is given by

$$
\left(R_{h}+\frac{W_{h}}{2}\right)^{2}=X_{h}^{2}+\left(Y_{h}-R_{h}\right)^{2}
$$

where $X_{h}, Y_{h}$ and $W_{h}$ are the longitudinal position, lateral position and width of the host vehicle.

Inserting (2) in (3) and solving for TTC $\triangleq\left|r_{x} / \dot{r}_{x}\right|$, gives

$$
\begin{aligned}
& a_{y_{h}}^{\mathrm{req}}=\frac{v_{x_{h}}^{2}\left(W_{h}+2 Y_{h}\right)}{X_{h}^{2}+Y_{h}^{2}-\frac{W_{h}^{2}}{4}}, \\
& \mathrm{TTC}=\left|\frac{X_{l}-X_{h}}{v_{x_{l}}-v_{x_{h}}}\right|,
\end{aligned}
$$

where $X_{l}$ and $v_{x_{l}}$ are the longitudinal position and speed of the leading vehicle, with $v_{x_{l}}<v_{x_{h}}$ and $r_{x}$ and $\dot{r}_{x}$ are the relative longitudinal distance and velocity between the vehicles, respectively. The detailed procedure of typical collision warning algorithm, which to the rest of the paper is referred to as CWA1, is presented in Algorithm 1. TTC is repeatedly computed with a predefined update rate, where in each update both $r_{x}$ and $\dot{r}_{x}$ are measured. If TTC has a finite value that is less than or equal to a predefined limit $\mathrm{TTC}_{\max }$, then $a_{y_{h}}$ and STN are also computed, and the algorithm issues a warning when STN exceeds a given threshold $T_{h_{1}}$.

CWA1 has two main limitations: i) it does not consider realistic constraints, such as the vehicle's dynamics or physi$\mathrm{cal} / \mathrm{road}$ limitations, and ii) the STN factor is compared with a speed-invariant threshold, which, as will be shown later, in Section V, may fail to give warning at certain safety-critical scenarios.

\section{THE NOVEL COLLISION WARNING ALGORITHM}

With respect to previous solutions [1], [12], [16], this work presents two major improvements: i) bicycle model dynamics and constraints are used in order to generate more accurate, reliable trajectories for lane change maneuver and ii) speeddependent threshold is defined that enables early warnings 

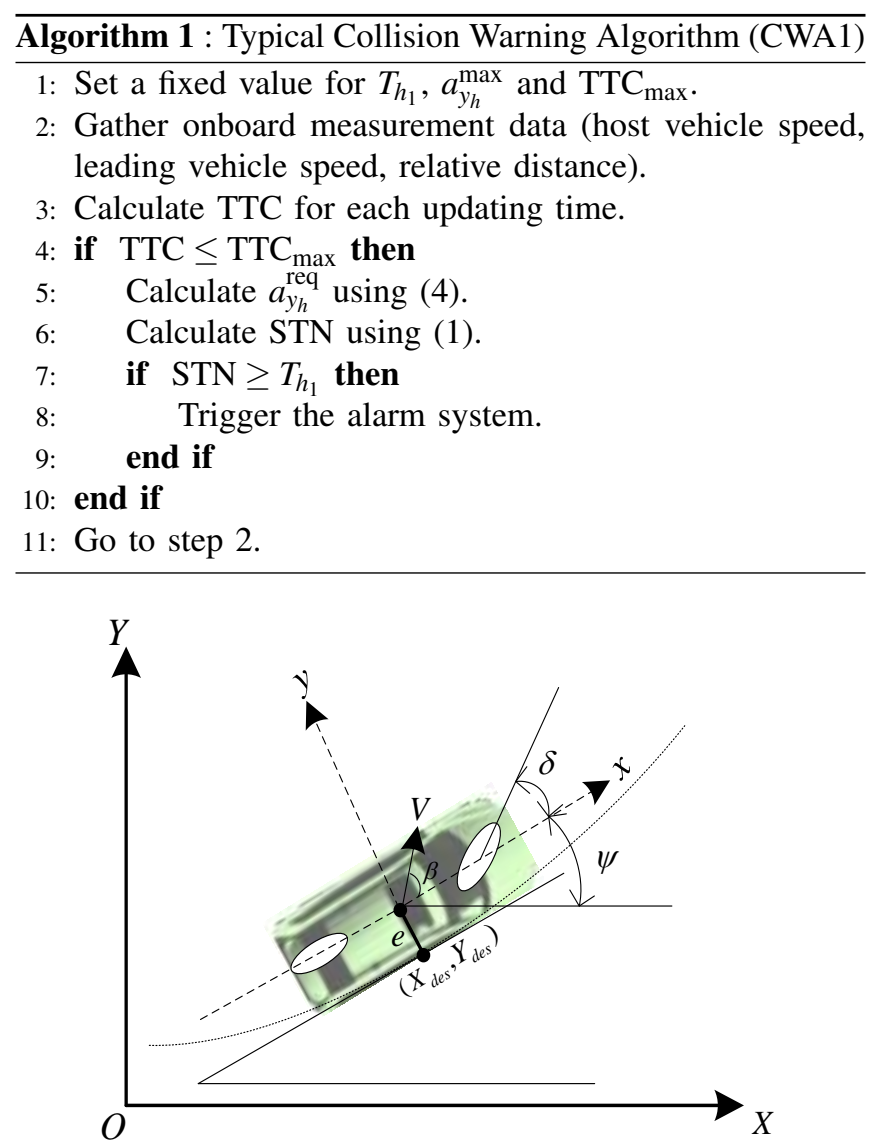

Fig. 3. Vehicle model in local and global coordinates.

adapted to the speed of the host and the leading vehicle. The proposed algorithm, which is referred to as CWA2, considers the following assumptions:

Assumption 1. The host vehicle longitudinal speed is constant during the lane change manoeuver. Furthermore, there are no vehicles in the adjacent lane in the neighbourhood of the leading vehicle.

Assumption 2. We assume that the leading vehicle moves along its path with zero lateral displacement and with a constant speed during the lane change maneuver.

Assumption 3. Threat is assessed when the TTC is a finite quantity. In other words, for cases when the host and the leading vehicle move with identical speeds, or there is no vehicle in front of the host vehicle, the algorithm is in standby.

\section{A. Requested lateral acceleration for a bicycle model}

This section provides a mathematical background for computing the requested lateral acceleration when representing the vehicle by a bicycle model. It provides road, acceleration and collision avoidance constraints, and introduces a predictive STN.

1) Vehicle modelling: A common practise in automotive applications is to model the vehicle with a three-degreeof-freedom bicycle model [19] operating in the linear tire

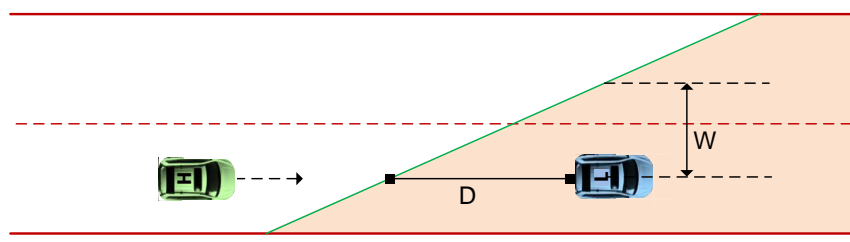

Fig. 4. Forward collision avoidance constraint which enforces the host vehicle from not entering the shaded area.

domain. In the global coordinate frame, it holds

$$
\dot{Y}_{h}(t)=v_{x_{h}} \sin \left(\psi_{h}(t)\right)+v_{y_{h}}(t) \cos \left(\psi_{h}(t)\right)
$$

where $Y_{h}$ and $v_{y_{h}}$ are lateral position and velocity, and $\psi_{h}$ is yaw angle. In the local, vehicle frame it holds

$$
\begin{aligned}
& \dot{z}_{h}(t)=A\left(v_{x_{h}}\right) z_{h}(t)+b \delta_{h}(t) \\
& a_{y_{h}}(t)=C\left(v_{x_{h}}\right) z(t)+d \delta_{h}(t) \\
& z_{h}(t)=\left[\begin{array}{lll}
v_{y_{h}}(t) & \dot{\psi}_{h}(t) & \psi_{h}(t)
\end{array}\right] \\
& A=\left[\begin{array}{ccc}
\frac{\alpha_{1}}{v_{x_{h}}} & -\left(v_{x_{h}}-\frac{\alpha_{2}}{v_{x_{h}}}\right) & 0 \\
\frac{\alpha_{3}}{v_{x_{h}}} & \frac{\alpha_{4}}{v_{x_{h}}} & 0 \\
0 & 1 & 0
\end{array}\right], \quad b=\left[\begin{array}{c}
\alpha_{5} \\
\alpha_{6} \\
0
\end{array}\right] \\
& C=\left[\begin{array}{ccc}
\frac{\alpha_{1}}{v_{x_{h}}} & \frac{\alpha_{2}}{v_{x_{h}}} & 0
\end{array}\right], \quad d=\alpha_{5}
\end{aligned}
$$

where $a_{y_{h}}$ is the lateral acceleration (output of the system), $\delta_{h}$ is the steering angle (input to the system) and $z_{h}$ is the state vector in the local coordinates. The coefficients, $\left\{\alpha_{i}\right\}_{i=1}^{6}$ are functions of the vehicle's parameters (mass, yaw moment of inertia, distance from center of gravity (c.g.) to front and rear tires, lateral tire force on front and rear tires) and are computed using small angle approximation, $\tan \left(\beta_{h}\right) \simeq \beta_{h}$, of the slip angle $\beta_{h}$, see [19]. An illustration of a vehicle in the global and local coordinate frame is depicted in Fig. 3.

2) Physical constraints: In order to guarantee a feasible lane change maneuver such that the host vehicle remains within the road limits and satisfies the actuator limitations, i.e., acceleration constraints, the following constraints are introduced

$$
\begin{aligned}
& Y_{\min } \leq Y_{h}(t) \leq Y_{\max }, \\
& v_{x_{h}} \beta_{\min } \leq v_{y_{h}}(t) \leq v_{x_{h}} \beta_{\max }, \\
& \left|a_{y_{h}}(t)\right| \leq a_{y_{h}}^{\max }
\end{aligned}
$$

where $Y_{\min }, Y_{\max }, \beta_{\min }$ and $\beta_{\max }$ are the minimum and maximum acceptable lateral displacements and body side slip angles, respectively. Note that the limits on the lateral velocity $v_{y_{h}}$ are enforced due to environmental conditions which can greatly influence the friction between tires and road surface.

3) Collision avoidance constraints: In order to achieve a safe lane change manoeuver, the host vehicle is kept at a certain distance from the leading vehicle. This constraint is formulated as a ramp barrier,

$$
\frac{r_{x}(t)}{D}+\frac{r_{y}(t)}{W} \geq 1,
$$


passing through the points defined by a certain longitudinal distance $D=v_{x_{h}} t_{d}+L_{l}$ from the leading vehicle, and desired lateral position $W=0.5 W_{L}+W_{l}$ at the end of the lane change maneuver, see Fig. 4. Here, $W_{l}$ and $L_{l}$ are the width and length of the leading vehicle, $t_{d}$ is the desired headway time and $W_{L}$ is lane width. The measures $r_{x}$ and $r_{y}=-Y_{h}$ are the relative longitudinal and lateral distances between the two vehicles.

4) Predictive steering threat number: Since the lateral acceleration of the presented bicycle model is not a constant, but rather a time-varying signal, we define a predictive steering threat number (PSTN),

$$
\operatorname{PSTN} \triangleq\left\|\frac{a_{y_{h}}(t)}{a_{y_{h}}^{\max }}\right\|_{\infty}
$$

that is a measure of the maximum STN from all time instances $t \in\left[0, t_{f}\right]$ along a predicted acceleration trajectory $a_{y_{h}}(t)$ within a receding horizon with size $t_{f}=$ TTC.

5) Optimization problem: Finally, after all ingredients have been presented, the optimization problem minimizing PSTN is formulated as

$$
\begin{aligned}
& \min _{\delta_{h}(t)} \text { PSTN } \\
& \text { subject to } \\
& \dot{Y}_{h}(t)=v_{x_{h}} \psi_{h}(t)+v_{y_{h}}(t) \\
& \dot{z}_{h}(t)=A\left(v_{x_{h}}\right) z_{h}(t)+b \delta_{h}(t) \\
& a_{y_{h}}(t)=C\left(v_{x_{h}}\right) z_{h}(t)+d \delta_{h}(t) \\
& Y_{\min } \leq Y_{h}(t) \leq Y_{\max } \\
& v_{x_{h}} \beta_{\min } \leq v_{y_{h}}(t) \leq v_{x_{h}} \beta_{\max } \\
& \frac{r_{x}(t)}{D}+\frac{r_{y}(t)}{W} \geq 1 \\
& \left|a_{y_{h}}(t)\right| \leq a_{y_{h}}^{\max } \\
& Y_{h}(0)=0, Y\left(t_{f}\right)=Y_{f} \\
& v_{y_{h}}(0)=0, v_{y_{h}}\left(t_{f}\right)=0 \\
& \dot{\psi}(0)=0, \dot{\psi}\left(t_{f}\right)=0 \\
& a_{y_{h}}(0)=0, a_{y_{h}}\left(t_{f}\right)=0
\end{aligned}
$$

where the constraints (13b)-(13h) are enforced for all $t \in\left[0, t_{f}\right]$, and a small angle approximation, in the lane change maneuver, is used

$$
\sin \left(\psi_{h}(t)\right) \cong \psi_{h}(t), \quad \cos \left(\psi_{h}(t)\right) \cong 1
$$

When written in discrete time, the problem (13) is a linear program, where control signal is the steering angle $\delta_{h}$.

Similar to the CWA1, the optimization problem (13) is repeatedly solved with a predefined update rate. For each update, the host vehicle speed is measured and the leading vehicle speed and inter vehicle distance are estimated using radar sensor system, the system matrices in (7) are computed, and feasible control and state trajectories are obtained by solving problem (13). The algorithm is summarized in Algorithm 2.

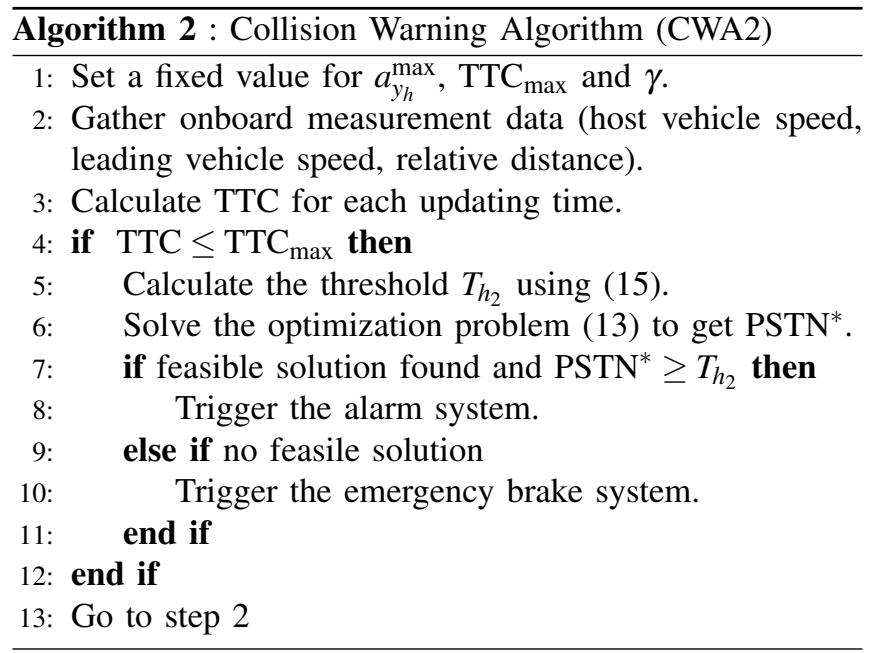

\section{B. Threshold design}

The warning system is triggered when the optimal PSTN (denoted as PSTN ${ }^{*}$ ) exceeds a threshold $T_{h_{2}}$. An appropriately selected threshold should guarantee an early warning such that the driver has enough time to respond, regardless of the speed of the host and leading vehicle. In this regard, some important aspects such as driver reaction time or brake system response time (delay) may be considered.

Analysis of real traffic data has shown that drivers are likely to initiate braking before TTC reaches 4-5 seconds [20]. Therefore, the CWA would need to be designed with a low threshold in order to give an earlier warning, i.e. to issue a warning at longer TTC.

However, a very low threshold (for instance at TTC of about 7-8 seconds) may also lead to a high percentage of false alarms [20], when, e.g., vehicles are travelling with low velocities.

Here, we present a threshold that adapts to vehicles speeds

$$
T_{h_{2}} \triangleq \gamma \sqrt{\frac{v_{x_{h}}}{v_{x_{h}}-v_{x_{l}}}} \leq 1,
$$

where $\gamma \leq 1$ is a scaling factor selected by the designer.

The idea behind the adaptive threshold is to employ the relation between the requested lateral acceleration and longitudinal speed [19]. From the definition of PSTN in (12) it can be observed that the numerator is proportional to the relative inter-vehicle speed, while the denominator depends only on the host vehicle speed. Thus, for an increased relative speed, PSTN will increase and $T_{h_{2}}$ decrease, which consequently enables an early warning.

\section{Simulation Results}

This section provides a case study by parameterizing CWA1 and CWA2 with the values given in Table I. The bicycle model (6)-(7) is parameterized for a Volvo S60, see [21].

The dependence of the STN index on the relative intervehicle distance is given in Fig. 5, for the case when CWA1 is employed. The markers on the figure lines denote different updates at which TTC is recomputed and collision threat is 




Fig. 5. Collision warning curves using CWA1 for three different speeds of the host vehicle. When the relative distance decreases, the value of the STN increases. The dots denote update intervals when TTC is re-computed. At the first update after the threshold is crossed, the driver will receive an alarm.

assessed. It can be noticed that the STN increases when the inter-vehicle distance decreases and when the host vehicle speed increases. The value for the leading vehicle speed is $v_{x_{l}}=17 \mathrm{~m} / \mathrm{s}$ and the host vehicle speed $\left(v_{x_{h}}\right)$ is 18 to $20 \mathrm{~m} / \mathrm{s}$. In all three cases of host vehicle speeds, the higher the speed is, the sooner the warning is triggered (when the green horizontal line, denoting the fixed threshold $T_{h_{1}}$, intersects with the STN curves).

The performances of CWA2 is analyzed in Fig. 6, in an identical setup. In addition to vehicles speed, the shapes of the PSTN curves depend also on the vehicle's dynamics, physical and collision avoidance constraints. The markers in the figure are obtained by re-solving problem (13) with the same update rate as with CWA1. The optimization problem (13) is written in CVX modeling language [22] and solved with SeDuMi [23]. It can be noticed that warning is issued long before PSTN reaches 1 , as a result of the adaptive threshold (the horizontal lines in Fig. 6). The highest point on the PSTN lines, which has to be $\leq 1$, depicts the last update before collision can be avoided by steering action. At the next update the problem is infeasible and the intervention system, e.g. emergency braking, should be activated.

The results of two algorithms, CWA1 and CWA2, are compared in Fig. 7. The two subplots show both STN and PSTN curves for two different speeds of the host vehicle.

TABLE I

SimUlation PARAMETERS.

\begin{tabular}{|l|l|l|}
\hline$a_{y_{h}}^{\max }=7\left[\mathrm{~m} / \mathrm{s}^{2}\right]$ & $Y_{f}=4[\mathrm{~m}]$ & $T_{h_{1}}=0.09$ \\
\hline$W_{L}=4[\mathrm{~m}]$ & $W_{l}=2[\mathrm{~m}]$ & $L_{l}=3.5[\mathrm{~m}]$ \\
\hline$t_{d}=0.2[\mathrm{~s}]$ & $Y_{\min }=-2.5[\mathrm{~m}]$ & $Y_{\max }=7[\mathrm{~m}]$ \\
\hline$\beta_{\min }=-0.2$ & $\beta_{\max }=0.2$ & $\gamma=0.028$ \\
\hline
\end{tabular}

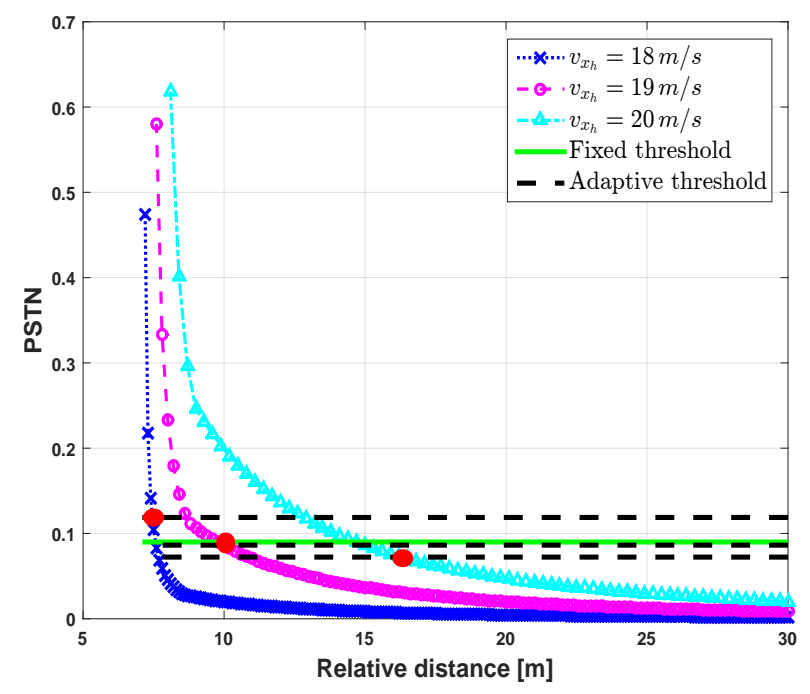

Fig. 6. Collision warning curve using CWA2 for three different speeds of the host vehicle. The higher the speed, the sooner a warning. The last point on the PSTN lines show that there exists a feasible solution to avoid collision. At the next update the problem is infeasible and the intervention system, e.g. emergency braking, should be activated.
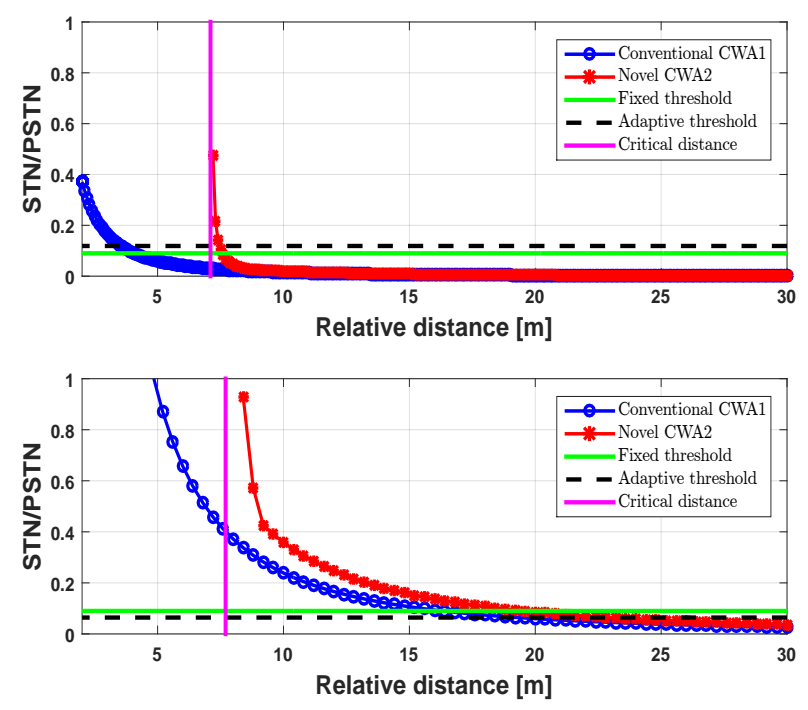

Fig. 7. STN curves for the CWA1 (blue lines) and PSTN curves for the CWA2 (red lines). The speed of the host vehicle is $18.5 \mathrm{~m} / \mathrm{s}$ and $21 \mathrm{~m} / \mathrm{s}$, in the top and bottom subplot, respectively. The speed of the leading vehicle is $17 \mathrm{~m} / \mathrm{s}$ in both subplots.

The update interval in both subplots is $0.1 \mathrm{~m}$. In both cases, the STN curves (for CWA1) reach critical values at shorter relative distances, while the PSTN curves (for CWA2) show that emergency braking is needed sooner, at longer relative distances. The magenta lines represent the critical distance $D$ that the host vehicle should not exceed. It can be seen that CWA1 (blue curve) fails to generate proper warnings (in the top subplot) while the CWA2 (red curve) alerts the driver properly. In both subplots, both the fixed and adaptive thresholds are depicted.

Finally, a comparison between the fixed threshold $T_{h_{1}}$ and adaptive threshold $T_{h_{2}}$ is provided in Fig. 8, when applied 


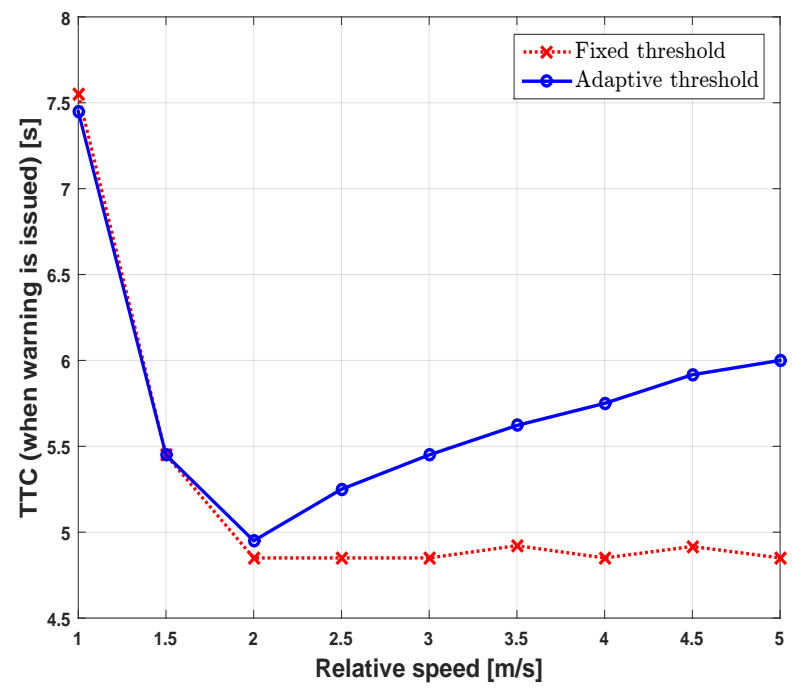

Fig. 8. TTC (when warning is issued) using both the fixed and adaptive thresholds.

to CWA2. The leading vehicle speed is $17 \mathrm{~m} / \mathrm{s}$ and the host vehicle speed varies from 18 to $22 \mathrm{~m} / \mathrm{s}$. It can be noticed that for low relative speeds, both thresholds have about the same warning time before the TTC. However, for relative speeds higher than $2 \mathrm{~m} / \mathrm{s}$ the adaptive threshold issues earlier warnings. Also, the TTC with the adaptive threshold is below 7 seconds, thus ensuring a low number of false alarms [20].

\section{CONCLUSIONS AND FUTURE WORK}

This paper shows a modified CWA which can generate a more accurate forward collision warning system. A convex optimization problem subject to vehicle's dynamics, physical and collision avoidance constraints is formulated to compute the predicted STN at all time instances until a potential lane change maneuver is completed. Regarding the previously presented methods [1], [12], [16] the proposed CWA is, in our opinion, more realistic. Moreover, an adaptive threshold for triggering the warning system is introduced. It considers both the host and leading vehicles speeds in order to produce earlier warnings, without increasing the number of false alarms.

Future studies may focus on extending the algorithm by combining steering and braking threat number. The inclusion of a detailed model for the human reaction time may be also subject to future studies. Furthermore, CVX will be bypassed by translating the optimization problem into corresponding C codes using, e.g. CVXGEN [24] tool. This will enable real-time usage of the proposed CWA.

\section{REFERENCES}

[1] J. Jansson, "Collision avoidance theory with application to automotive collision mitigation," Ph.D. dissertation, Department of Electrical Engineering,Linköping University, Sweden, 2005.

[2] N. Augustsson and M. Wilhelmsson, "Verification of collision avoidance functionality a mileage multiplier approach to verify future collision avoidance systems," Master's thesis, Chalmers University of Technology, Gothenburg, Sweden, 2010.
[3] J. Alonso, E. Vidal, A. Rotter, and M. Mühlenberg, "Lane-change decision aid system based on motion-driven vehicle tracking," IEEE Transactions on Vehicular Technology, vol. 57, no. 5, pp. 2736-2746, 2008.

[4] P. Barber and N. Clarke, "Advanced collision warning systems," in IEEE Colloquium on Industrial Automation and Control: Applications in the Automotive Industry, 2/1, 2/9, 1998.

[5] A. Burgett, A. Carter, R. Miller, W. Najm, and D. Smith, "A collision warning algorithm for rear-end collisions," in 16th International Techical Conference on Enhanced Safety of Vehicles, 566-587, 1998.

[6] C. Eberhard and D. Moffa, "Collision warning," Automotive Engineering, vol. 105, no. 3, p. 8690, 1997.

[7] B. Chen, C. Shih, and Y.lin, "Design of forward collision warning system using estimated relative acceleration and velocity vector,' SAE International, Technical Paper 2014-01-2030, 2014.

[8] A. Polychronopoulos, M. Tsogas, A. Amditis, U. Scheunert, L. Andreone, and F. Tango, "Dynamic situation and threat assessment for collision warning systems," in IEEE Intelli-gent Vehicles Symposium, Parma, Italy, 2004.

[9] J. Hillenbrand, A. Spieker, and K. Kristian, "A multilevel collision mitigation approach - its situation assessment, decision making and performance tradeoffs," IEEE Transactions on Intelligent Transportation Systems, vol. 7, no. 4, pp. 528-540, 2006.

[10] T. Shim, G. Adireddy, and H. Yuan, "Autonomous vehicle collision avoidance system using path planning and model predictive control based active front steering and wheel torque control," Proc. of the Institution of Mechanical Engineers, Part D: Journal of Automobile Engineering June, vol. 226, no. 4, pp. 767-778, 2012.

[11] D. Soudbakhsh, A. Eskandarian, and D. Chichka, "Vehicle collision avoidance maneuvers with limited lateral acceleration using optimal trajectory control," Journal of Dynamic Systems, Measurement, and Control, vol. 135, no. 5, pp. 1-12, 2013.

[12] J. Nilsson, M. Ali, P. Falcone, and J. Sjöberg, "Receding Horizon Manoeuvre Generation for Automated Highway Driving," Control Engineering Practice, vol. 41, pp. 124-133, 2015.

[13] Z. Shiller and S. Sundar, "Emergency lane-change maneuvers of autonomous vehicles," Journal of Dynamic Systems, Measurement, and Control, vol. 120, no. 5, pp. 37-44, 1998.

[14] G. P. Bevan, H. Gollee, and J. Reilly, "Trajectory generation for road vehicle obstacle avoidance using convex optimization," in Proc. Institue of Mechanical Engineering, vol. 224, 2010, pp. 455-473.

[15] M. Brännström, J. Sjöberg, and E. Coelingh, "A situation and threat assessment algorithm for a rear-end collision avoidance system," in IEEE Intelligent Vehicles Symposium Eindhoven University of Technology Eindhoven, Netherlands, 2008.

[16] J. Sjöberg, E. Coelingh, M. Ali, M. Brännström, and P. Falcone, "Driver models to increase the potential of automotive active safety functions," in 18th European Signal Processing Confrence, Denmark, 2010.

[17] J. Jansson and W. Birk, "An alternative brake criterion for collision avoidance," US Patent Application Publication, US2005/0090955A1, National Renewable Energy Laboratory, 2006.

[18] G. Engelman, J. Ekmark, L. Tellis, M. Tarabishy, G. Joh, R. Trombley, and R. Williams, "Threat level identification and quantifying system," US Patent Application Publication, US2005/0090955A1, National Renewable Energy Laboratory, 2005.

[19] R. Rajamani, Vehicle Dynamics and Control, 2nd ed. Springer, 2012.

[20] B. Sultan and M. McDonald, "Assessing the safety benefit of automatic collision avoidance systems," in 18th International Technical Conference on the Enhanced Safety of Vehicles (ESV), Washington, 2003.

[21] A. Dahlin, A. Larsen, E. Pearson, J. Brickner, M. Herna, and R. Akerlund, "Testing active safety systems development of an experimental platform for cooperative driving and collision avoidance strategies," Master's thesis, Chalmers University of Technology, Gothenburg, Sweden, 2015.

[22] M. Grant and S. Boyd, "CVX: Matlab software for disciplined convex programming, version 2.1," http://cvxr.com/cvx, Mar. 2014.

[23] J. Sturm, "Using sedumi 1.02, a matlab toolbox for optimization over symmetric cones," Optimization Methods and Software, vol. 11-12, 1999.

[24] J. Mattingley and S. Boyd, "Using sedumi 1.02, a matlab toolbox for optimization over symmetric cones," Optimization Methods and Software, vol. 13, 2012. 